\title{
Efeitos das ondas de choque piezoelétrica na adiposidade localizada
}

\author{
Effects of piezoelectric shock waves on localized adiposity
}

\section{Rodrigo Marcel Valentim da Silva \\ Fábio dos Santos Borges ${ }^{2}$ \\ Júlio Davi Costa e Silva ${ }^{3}$ \\ Jéssica Carrie Silva de Oliveira Lira ${ }^{4}$ \\ Maria Paula Marques de Oliveira ${ }^{5}$ \\ Louhanne Christine Berto Bezerra ${ }^{6}$}

Bruna Nobrega Bezerra

Patrícia Froes Meyer ${ }^{8}$

Endereço para Correspondência: Rodrigo Marcel Valentim da Silva Rua Nossa Senhora de Fátima, 312 b - Alecrim 59030-080 - Natal - RN [Brasil] marcelvalentim@hotmail.com

\begin{abstract}
Resumo
Introdução: A terapia de ondas de choque piezoelétrica (TOC) é uma técnica não invasiva que visa a reduzir o tecido adiposo subcutâneo localizado. Objetivos: Investigar os efeitos da terapia ondas de choque piezoelétrica na redução da adiposidade subcutânea localizada. Métodos: Efetuou-se um estudo experimental, com 31 participantes, de 25 a 45 anos, do sexo feminino. Foram realizadas quatro sessões de tratamento, uma vez por semana com parâmetros da OC piezoelétrica. Procedeu-se à aplicação dinâmica na região infraumbilical; e estacionária, na supraumbilical, ambas no lado esquerdo. O lado direito não recebeu aplicação, tornando-se o lado controle. Resultados: Na aplicação da TOC piezoelétrica na forma estacionária, houve maior redução de tecido adiposo do que no modo dinâmico. Conclusão: Pode-se observar que a terapia por OC piezoelétrica promoveu uma redução da adiposidade localizada, com o modelo de equipamento escolhido e na dose proposta neste estudo, constatada nas análises de plicometria e ultrassonografia.
\end{abstract}

Descritores: Gordura. Fisioterapia. Transdutores. Modalidades de fisioterapia.

FATERN. Natal, RN - Brasil.

ORCID: https://orcid.org/0000-0002-5859-4599

2 Departamento de Fisioterapia da Faculdade Estácio de Sá Estácio/RJ. Rio de Janeiro, RJ - Brasil.

ORCID: https://orcid.org/0000-0002-9440-9127

3 Departamento de Fisioterapia da Universidade Potiguar UNP. Natal, RN - Brasil.

ORCID: https://orcid.org/0000-0001-5131-0285

4 Departamento de Fisioterapia do Centro Universitário do Rio Grande do Norte - UNI-RN. Natal, RN - Brasil. ORCID: https://orcid.org/0000-0002-5631-5592

5 Departamento de Fisioterapia da Universidade Potiguar UNP. Natal, RN - Brasil.

ORCID: https://orcid.org/0000-0002-5208-2912

6 Departamento de Fisioterapia da Universidade Potiguar UNP. Natal, RN - Brasil.

ORCID: https://orcid.org/0000-0002-1141-5979

7 Departamento de Fisioterapia da Universidade Potiguar UNP. Natal, RN - Brasil.

ORCID: https://orcid.org/0000-0002-5285-3262

8 Departamento de Fisioterapia da Universidade Potiguar UNP. Natal, RN - Brasil.

ORCID: https://orcid.org/0000-0003-4922-8092

\begin{abstract}
Introduction: Piezoelectric shock wave therapy (PSWT) is a noninvasive technique that aims to reduce localized subcutaneous adipose tissue. Objectives: To investigate the effects of piezoelectric shock wave therapy on the reduction of localized subcutaneous adiposity. Methods: An experimental study, with 31 female participants, aged between 25 and 45 years, was conducted. Four treatment sessions were performed once a week with different parameters of the piezoelectric SW. They were applied dynamically in the infraumbilical area, and stationarily in the supraumbilical area, both on the left side. The right side did not receive therapy, becoming the control side. Results: In the application of piezoelectric SWs in stationarily, there was a greater reduction of adipose tissue than in dynamic mode. Conclusion: The therapy by piezoelectric SWs promoted a reduction of the localized adiposity, with the model of equipment chosen and at the dose proposed in this study, verified through the analysis of plicometry and ultrasonography data.
\end{abstract}

Keywords: Fats. Physical therapy. Transducers. Physical therapy modalities. 


\section{Introdução}

A terapia por ondas de choque (TOC) tem sido largamente utilizada no processo de reparo e regeneração de tecidos, no alívio de dor e nas diferentes intervenções estéticas, entre essas, a redução da adiposidade localizada. A TOC se caracteriza por um estímulo mecânico criado a partir de fontes eletromagnéticas, biomecânicas ou pneumáticas ${ }^{1}$. As ondas de choque, do ponto de vista da física, não são ondas elétricas, mas sim impulsos acústicos/mecânicos de características definidas por uma variação brusca de pressão, seguida por rápido decréscimo chegando a pressões negativas ${ }^{1,2}$. Este gradiente de pressão muito rápido (medido em nanosegundos) e de alta frequência causa nos tecidos um fenômeno denominado cavitação, gerado a partir da ocorrência de microbolhas no meio de sua propagação. O impacto mecânico e a eclosão destas microbolhas promovem uma série de alterações, que depende diretamente da intensidade da força que estas ondas possuem ao atingir o tecido a ser tratado ${ }^{2,3}$.

Dentre os diferentes efeitos fisiológicos promovidos pela TOC pode-se destacar um mecanismo que induz a liberação de fatores de crescimento, bem como aumento da circulação local. Esse mecanismo promove um aumento do aporte de nutrientes no tecido e proporciona a diminuição de áreas isquêmicas ${ }^{4-6}$.

Atualmente, a TOC tem sido utilizada na área da estética para tratar alterações, tais como flacidez, fibroedemageloide e adiposidade localizada. É capaz de induzir respostas intersticiais e extracelulares, estimulando reparo e regeneração dos tecidos. Esta é uma modalidade de tratamento que tem a vantagem de não ser invasiva e não apresentar riscos cirúrgicos, podendo ser realizada em ambiente ambulatorial ${ }^{6}$.

A aplicação da TOC promove a liberação de energia produzida no local a tratar. A TOC pode ser dividida em três categorias, a saber: baixa energia $\left(<0,08 \mathrm{~mJ} / \mathrm{mm}^{2}\right)$, energia média $\left(0,08-0,28 \mathrm{~mJ} / \mathrm{mm}^{2}\right)$ e alta energia $(>0,28 \mathrm{~mJ} /$ $\left.\mathrm{mm}^{2}\right)$, esta última é usada no tratamento de al- terações subcutâneas, como a adiposidade localizada. Quanto à quantidade de pulsos a serem utilizados, não há um número de pulsos específico predeterminado. Contudo, de acordo com alguns estudos, a aplicação de TOC, com variação entre 1500 e 2000 pulsos, proporciona efeitos satisfatórios nos tecidos tratados ${ }^{5-7}$.

A TOC pode estar associada a diferentes tipos de fontes emissoras, com um sistema: eletro-hidráulico, eletromagnético e piezoelétrico. O princípio eletro-hidráulico refere-se a ondas acústicas de alta energia geradas pela explosão subaquática com descarga de centelha de eletrodo de alta tensão. Essas ondas são focadas com um refletor elíptico e direcionadas para a área que precisa de reparo, a fim de produzir efeito terapêutico. O princípio eletromagnético envolve a corrente elétrica que passa através de uma bobina para produzir um campo magnético forte, uma lente é usada para focar as ondas, sendo o ponto focal definido pelo comprimento da lente. O princípio piezoelétrico envolve um grande número de piezocristais montados em uma esfera - geralmente maior que mil cristais -, e recebe uma descarga elétrica rápida que induz um pulso de pressão na inclinação da água circundante para uma onda de choque. Os arranjos dos cristais causam autofoco das ondas em direção ao centro-alvo e levam a uma focagem extremamente precisa e de alta energia dentro de um volume focal definido. Sendo assim, a equipamento piezoelétrico promove a focalização da energia, de modo que se acredita que, no tecido subcutâneo, esse estímulo promoveria uma resposta ao nível do tecido adiposo, o que favoreceria alterações metabólicas nesse tecido ${ }^{7,8}$.

As propriedades específicas das OC piezoelétricas e sua relação com o tratamento da adiposidade localizada ainda não estão bem descritas na literatura, necessitando de análises consistentes quanto aos seus efeitos. Portanto, o objetivo principal deste estudo foi investigar os efeitos das ondas de choque piezoelétricas no tratamento da adiposidade localizada em região abdominal de mulheres. 


\section{Metodologia}

Este estudo, de caráter experimental, foi realizado na Clínica Biofisio, em Natal, $\mathrm{RN}$, sendo submetido à apreciação do Comitê de Ética em Pesquisa da Universidade Potiguar - UnP (CEP-UNP), por meio da interface com a plataforma Brasil, e aprovado com número de protocolo 2.696.690. Neste trabalho, foram respeitados os aspectos éticos baseados na Resolução 453/2012 do Conselho Nacional de Saúde e na Declaração de Helsinki. Todos os voluntários concordaram em participar voluntariamente do estudo e assinaram o Termo de Consentimento Livre e Esclarecido - TCLE, que apresentava as informações sobre os propósitos, riscos e benefícios da pesquisa.

Participaram deste estudo, 31 mulheres, faixa etária de 25 a 45 anos, que apresentavam gordura subcutânea localizada na região infraumbilical. Foram exigidos os seguintes critérios de inclusão: mulheres sedentárias, que não estivessem fazendo dieta, que não tivessem sido acometidas de doenças metabólicas graves, que apresentassem o Índice de Massa Corporal (IMC) entre 18,5 e 29,99 (normal a sobrepeso), que tivessem gordura localizada infraumbilical maior que $15 \mathrm{~mm}$ (identificados via imagem ultrassonográfica) e que não estivessem fazendo uso de medicamentos com efeitos lipolíticos e/ ou termogênicos até uma semana antes do início do estudo. Foi orientado ainda que, caso alguma das voluntárias tivesse necessidade de fazer uso deste tipo de medicamento, após o início da pesquisa, seria descartada da amostra, fato que não ocorreu neste estudo.

As voluntárias foram avaliadas duas vezes durante a realização do estudo: antes e no fim do tratamento, utilizando-se, para isso, as medidas de perimetria, plicometria fotografias, peso corporal, análise ultrassonográfica e questionário de satisfação e reações ao tratamento.

Para a realização da perimetria, usou-se uma fita métrica posicionada a $3 \mathrm{~cm}$ abaixo da cicatriz umbilical, estando as voluntárias na posição em pé. Com posicionamento similar, foi realizada a plicometria nas regiões supraumbilical dos lados direito e esquerdo. A fita métrica retrátil utilizada foi a da marca Fiber ${ }^{\circledR}$, confeccionada em material plástico, medindo $100 \mathrm{~cm}$ de comprimento total.

Para a plicometria, foram efetuadas três medições em cada região a partir da prega cutânea em que foi feita a média dessas medidas. A plicometria foi realizada com um plicômetro da marca Sanny que apresenta um campo de medição de 0 a $65 \mathrm{~mm}$. Foi tomada a prega cutânea por três vezes, e o resultado foi baseado na média dos valores obtidos nas três mensurações. Os procedimentos de perimetria e plicometria foram feitos pelo mesmo avaliador.

Com as voluntárias em pé, foram colhidas três imagens fotográficas - de frente, perfil esquerdo e perfil direito -, utilizando-se o equipamento modelo Galaxy J5 Prime, fabricado pela Samsung. Usou-se, ainda, um fundo de cor neutra para obtenção padronizada das imagens.

A mensuração do peso corporal foi feita com a voluntária em pé, sobre a balança, com uso de roupa íntima. Foi utilizada uma balança digital, da marca Sanny®.

Foi usado o equipamento de ultrassom de imagem MSLPU35 Linear Wireless Probe Ultrasound Scanner $(10 \mathrm{MHz})$, fabricado pela Guangzhou Medsinglong Medical Equipment Co. Para a ultrassonografia, foram realizadas marcações $5 \mathrm{~cm}$ acima da cicatriz umbilical para análise da parte supraumbilical, e $5 \mathrm{~cm}$ abaixo da cicatriz para análise de infraumbilical (Figura 1-A).

As regiões de mensuração foram demarcadas com a voluntária em pé e o exame ultrassonográfico foi realizado com a participante em decúbito dorsal. O exame de ultrassonografia (US) foi feito por um médico especialista para análise e mensuração de tecido adiposo. O transdutor foi posicionado perpendicularmente ao tecido cutâneo, sem pressão sobre a pele para que não houvesse influências sobre o resultado. 

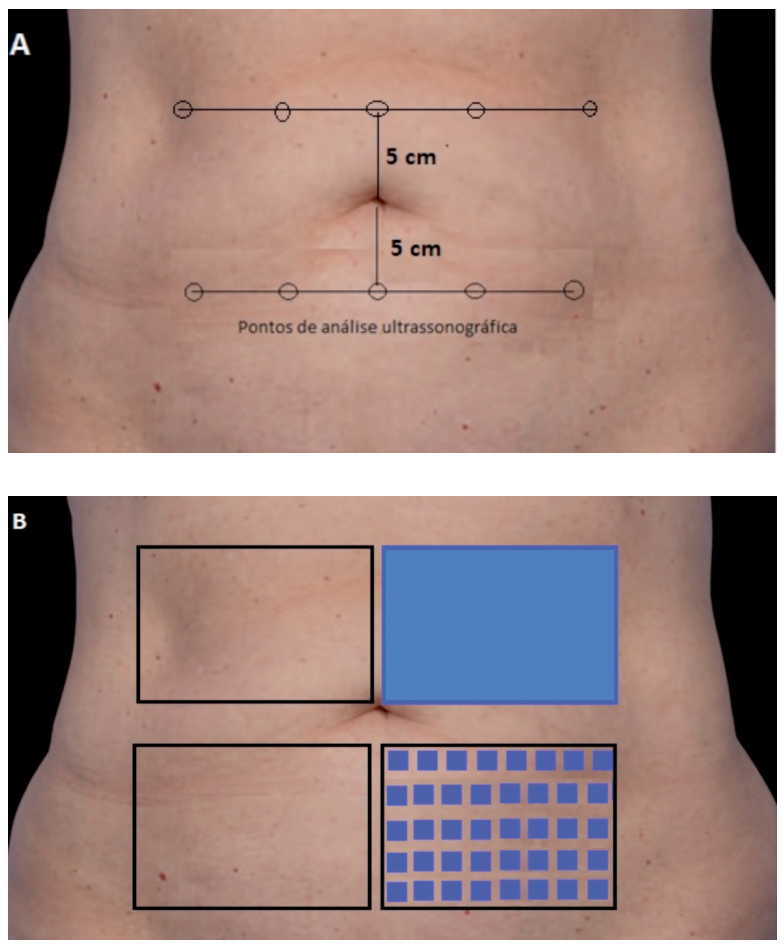

Figura 1: Demonstração da marcação das subáreas para realização do exame e tratamento. A: definição das zonas de anólise da ultrassonografia; B: zona infraumbilical, lado direito controle e lado esquerdo tratado com aplicação estacionória; e zona supraumbilical, lado direito controle e lado esquerdo tratado com aplicação dinâmica. Fonte: Os autores.

\section{Intervenção}

A região abdominal recebeu marcação apropriada para delimitação do local geral de tratamento e, após isso, foi dividida em quatro subáreas específicas, medindo 10 x 15 cm (150 $\mathrm{cm}^{2}$ ) (Figura 1-B).

As voluntárias foram tratadas com ondas de choque piezoelétrica na região supra e infraumbilical, totalizando quatro aplicações, com intervalo semanal entre elas.

Cada subárea foi tratada por dez minutos utilizando-se o lado direito como controle e o esquerdo, como tratado. Na região supraumbilical esquerda, foi feita a aplicação dinâmica; e na infraumbilical esquerda, a estacionária. No lado controle, não foi efetuada nenhuma intervenção.
A região do modo estacionário foi marcada com 40 pontos equidistantes (Figura 1-B), e o transdutor ficou imóvel sobre cada um dos pontos por cerca de 10 a 15 segundos de acordo com o nível de tolerância de cada voluntária tratada, durante o tempo previsto para a aplicação (10 min). No lado esquerdo, o transdutor foi utilizado com movimentos lentos abrangendo a subárea designada para a aplicação, durante 10 minutos (Figura 2).
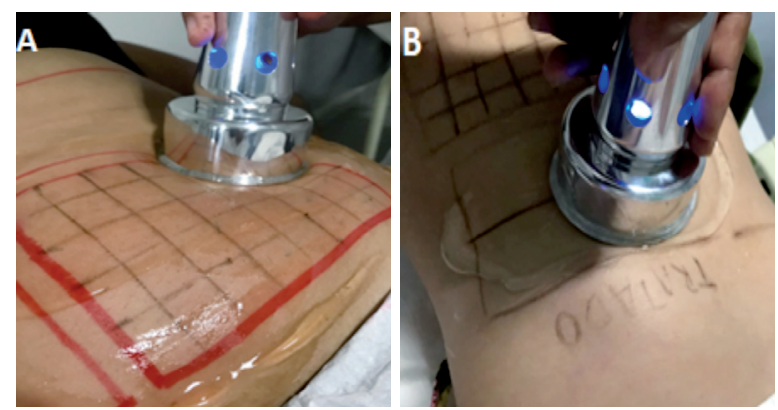

Figura 2: Tratamento da região abdominal. A: aplicação da onda de choque piezoelétrica na forma estacionória. B: aplicação da onda de choque piezoelétrica na forma dinômica

Fonte: Os autores.

Usou-se o equipamento de ondas de choque Lipocavity Wave, fabricado pela Medical San, arquitetado com cerâmica piezoelétrica minimamente côncava $(5 \mathrm{~mm})$ e profundidade da zona focal de ação de $4,5 \mathrm{~cm}$. Segundo, a empresa as doses variam entre 0,32 e $0,60 \mathrm{~mJ} / \mathrm{mm}^{2}$. Foi sugerido o protocolo de dose mais alta em ambos os modos de aplicação $\left(0,60 \mathrm{~mJ} / \mathrm{mm}^{2}\right)$, frequência de $15 \mathrm{~Hz}$.

\section{Reavaliação}

Após duas semanas do término do tratamento, as voluntárias foram reavaliadas empregando-se a mesma metodologia usada na avaliação inicial.

Além da reavaliação física local, as participantes responderam a um questionário de satisfação adaptado de Segot-Chicq et al. ${ }^{9}$ e um 
questionário relatando a ocorrência de possíveis efeitos adversos e/ou deletérios ocorridos durante e após o tratamento. Os efeitos adversos previstos neste questionário foram: dor (de leve a severa), dormência ou diminuição da sensibilidade, hipersensibilidade local, edemas/inchaços/bolhas localizadas transitórios, eritema (vermelhidão) após sessão, equimose/hematoma temporário, queimaduras, crescimento anormal de gordura no local do tratamento, outros efeitos adversos assim como a necessidade do auxílio médico para resolver alguma das adversidades.

A estatística descritiva e inferencial dos dados foi realizada por meio do programa SPSS 22.0 (Statistical Package for the Social Science, version 22.0). A normalidade na distribuição dos dados foi observada pelo teste de KolmogorovSmirnov (KS). Na análise descritiva, utilizou-se a média como medida de tendência central; e o desvio-padrão, como medida de dispersão.

Para a realização das comparações foi utilizado o teste $\mathrm{t}$ pareado. Para todas as análises estatísticas foi adotado um intervalo de confiança de $95 \%$ (IC 95\%).

\section{Resultados}

A Tabela 1 apresenta os resultados da caracterização antropométrica da amostra estudada.

Tabela 1: Análise descritiva e medidas de dispersão da população estudada

\begin{tabular}{l|c|c|c|c|c}
\hline & $\mathrm{N}$ & Média & $\begin{array}{c}\text { Desvio- } \\
\text { padrão }\end{array}$ & Mínimo & Máximo \\
\hline $\begin{array}{l}\text { ldade } \\
\text { (anos) }\end{array}$ & 31 & 26,45 & 5,98 & 19,00 & 43,00 \\
\hline $\begin{array}{l}\text { Peso } \\
(\mathrm{kg})\end{array}$ & 31 & 61,60 & 11,26 & 47,40 & 68,70 \\
\hline $\begin{array}{l}\mathrm{IMC} \\
\left(\mathrm{kg} / \mathrm{cm}^{2}\right)\end{array}$ & 31 & 23,92 & 4,27 & 21,00 & 24,05 \\
\hline
\end{tabular}

Fonte: Os autores.

A Tabela 2 apresenta a análise inferencial entre os momentos inicial e final das variáveis.
Tabela 2: Comparação entre as médias e desvio-padrão das varióveis peso, perimetria, plicometria direita, plicometria esquerda, US (ultrassonografia) supra e infraumbilical direita e esquerda

\begin{tabular}{l|c|c|c}
\hline & Inicial & Final & P valor \\
\hline Peso (kg) & $70,98 \pm 12,96$ & $73,96 \pm 15,54$ & 0,32 \\
\hline Perimetria (cm) & $80,5 \pm 7,03$ & $81,62 \pm 8,75$ & 0,39 \\
\hline $\begin{array}{l}\text { Plicometria } \\
\text { direita (cm) }\end{array}$ & $3,39 \pm 0,53$ & $3,32 \pm 0,39$ & 0,11 \\
\hline $\begin{array}{l}\text { Plicometria } \\
\text { esquerda (cm) }\end{array}$ & $3,29 \pm 0,51$ & $2,99 \pm 0,38$ & $0,006^{*}$ \\
\hline $\begin{array}{l}\text { US supra direita } \\
\text { (cm) }\end{array}$ & $1,75 \pm 0,77$ & $1,61 \pm 0,75$ & $0,001^{*}$ \\
\hline $\begin{array}{l}\text { US supra } \\
\text { esquerda (cm) }\end{array}$ & $1,68 \pm 0,74$ & $1,47 \pm 0,71$ & $0,001^{*}$ \\
\hline $\begin{array}{l}\text { US infra direita } \\
\text { (cm) }\end{array}$ & $2,37 \pm 0,87$ & $2,12 \pm 0,88$ & $0,001^{*}$ \\
\hline $\begin{array}{l}\text { US infra } \\
\text { esquerda (cm) }\end{array}$ & $2,40 \pm 0,90$ & $2,00 \pm 0,84$ & $0,001^{*}$ \\
\hline
\end{tabular}

${ }^{*} p<0,05$ = diferença significativa.

Fonte: Os autores.

Observou-se que as variáveis plicometria esquerda, US supra direita, US supra esquerda, US infra direita e US infra esquerda apresentaram resultados significativos com redução das medidas analisadas.

A Tabela 3 apresenta as comparações entre o lado controle (direito) e tratado (esquerdo), observou-se que o lado esquerdo apresentou uma redução significativa, comparado ao direito, nas medidas de plicometria, ultrassonografia supraumbilical e infraumbilical.

A Tabela 4 representa a comparação entre os efeitos obtidos nas regiões supraumbilical e infraumbilical esquerdas, que correspondem às regiões de aplicação dinâmica e estacionárias.

Verificou-se que, no momento inicial nas avaliações das medidas supra e infraumbilical, já existia uma diferença, fato que dificultou a interpretação dos resultados na avaliação final, quando também se observou uma diferença entre as zonas supra e infraumbilical esquerda. Mas ao analisar as imagens de ultrassonografia individualmente, é possível perceber a diferença entre as regiões tratadas e controle, conforme demonstra as Figuras 3A e 3B (análises ultras- 
Tabela 3: Anólise comparativa da região controle (direito) e da região tratada (esquerda)

\begin{tabular}{l|c|c}
\hline & p valor Inicial & p valor Final \\
\hline $\begin{array}{l}\text { PlicometriaDir x } \\
\text { PlicometriaEsq }\end{array}$ & 0,09 & $0,04^{*}$ \\
\hline $\begin{array}{l}\text { US Supra Dir x US } \\
\text { Supra Esq }\end{array}$ & 0,12 & $0,001^{*}$ \\
\hline $\begin{array}{l}\text { US Infra Dir X US } \\
\text { Infra Esq }\end{array}$ & 0,89 & $0,001^{*}$ \\
\hline
\end{tabular}

PlicometriaDir: plicometria direita, PlicometriaEsq: plicometria esquerda; US Supra Dir: ultrassonografia supraumbilical lado direito; US Supra Esq: ultrassonografia supraumbilical lado esquerdo, US Infra Dir: ultrassonografia infraumbilical lado direito; US Infra Esq: ultrassonografia infraumbilical lado esquerdo ${ }^{*} p<0,05=$ diferença significativa Fonte: Os autores.

Tabela 4: Comparação dos efeitos obtidos com a aplicação dinômica (supra) e estacionária (infra)

\begin{tabular}{l|c|c}
\hline & p valor Inicial & p valor Final \\
\hline $\begin{array}{l}\text { US Supra Ex US } \\
\text { Infra E }\end{array}$ & $0,001^{*}$ & $0,001 *$ \\
\hline
\end{tabular}

US Supra E: ultrassonografia na região supraumbilical esquerda; US Infra E: ultrassonografia na região infraumbilical esquerda

${ }^{*} p<0,05=$ diferença significativa

Fonte: Os autores.

sonográficas da região infra e supraumbilical esquerda antes do tratamento), Figuras $4 \mathrm{~A}$ e $4 \mathrm{~B}$ (análises ultrassonográficas da região infra e supraumbilical direita, controle), Figuras 5A e 5B (análises ultrassonográficas da região infra e supraumbilical esquerda após tratamento) e Figuras 6A e 6B (análises ultrassonográficas da região infra e supraumbilical direita após tratamento, controle).

Por meio das imagens de ultrassonografia demonstradas nas Figuras 3 e 5 e dos dados contidos na Tabela 2, verificou-se que a região tratada teve uma redução importante, comparada com a do lado controle, de $0,6 \mathrm{~cm}$ na região infraumbilical e $0,15 \mathrm{~cm}$ na região supraumbilical. Do total da amostra, 21 mulheres apresentaram esse comportamento, assim a diferença sempre foi mais acentuada na região infraumbilical, na qual o aplicador da TOC foi utiliza-

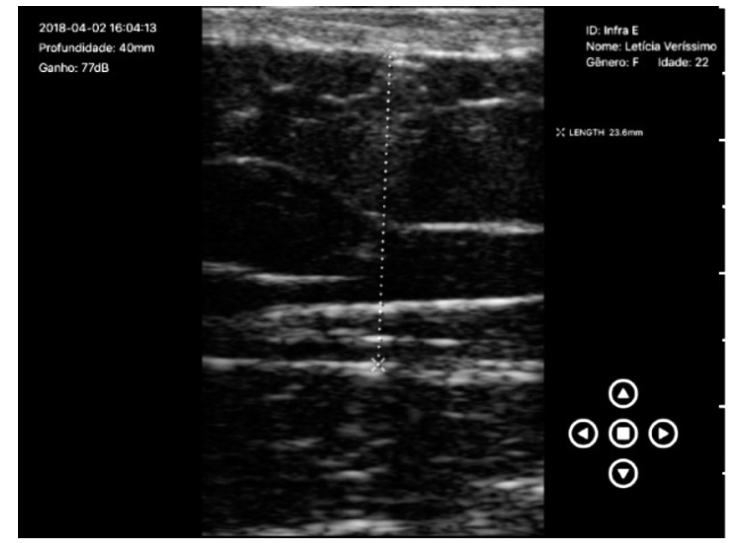

(A)

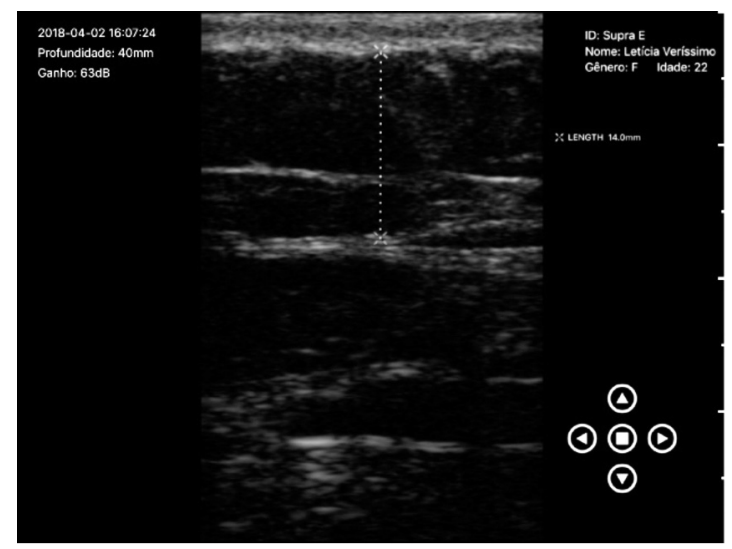

(B)

Figura 3: (A) Anólise ultrassonográfica da região infraumbilical esquerda, 2,36 cm; (B) Anólise ultrassonográfica da região supraumbilical esquerda antes do tratamento, $1,40 \mathrm{~cm}$

Fonte: Os autores.

do de forma estacionária. Mas no lado controle, percebeu-se que a região infraumbilical não se alterou após o período de quatro semanas e na região supraumbilical a redução foi $0,14 \mathrm{~cm}$, em uma voluntária. Em seis participantes, a região supraumbilical não apresentou redução. Não foram feitas análises em quatro mulheres, pois estas não realizaram o exame final. A análise foi feita pela avaliação do ultrassonografista.

A Tabela 5 apresenta os resultados referentes ao questionário de satisfação após o tratamento.

Observou-se, de acordo com o questionário de satisfação adaptado de Segot-Chicq, 2007, que, no relato das voluntárias, $12 \%$ perceberam 


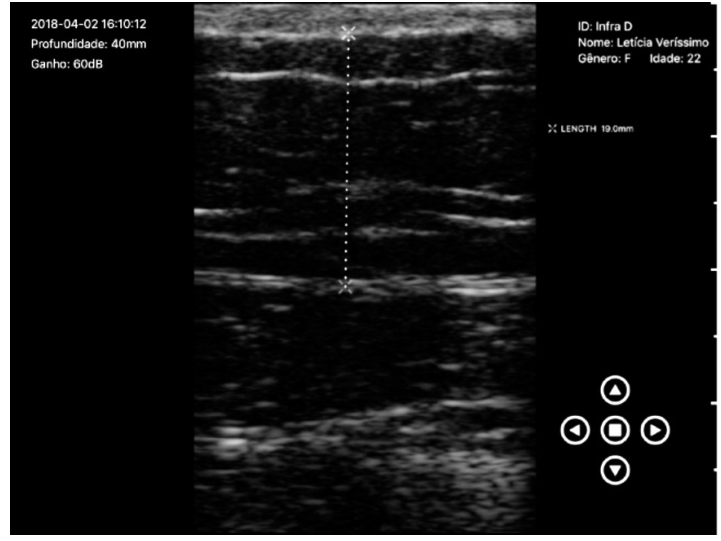

(A)

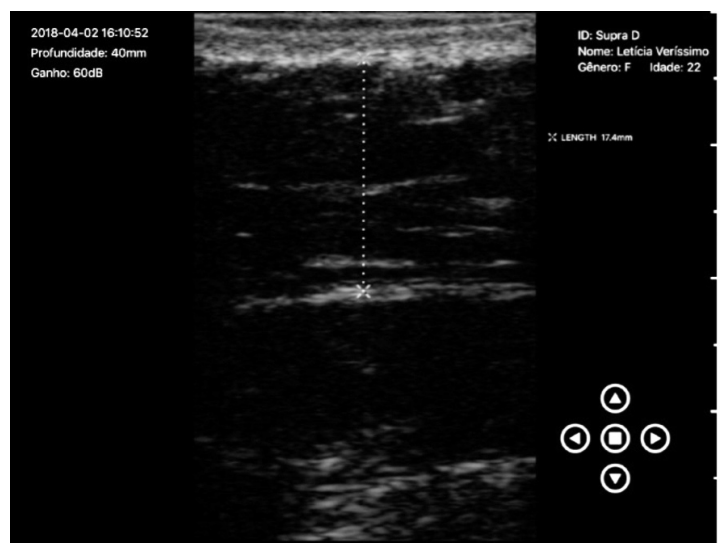

(B)

Figura 4: (A) Anólise ultrassonográfica da região infraumbilical direita antes do tratamento, lado controle, $1,90 \mathrm{~cm}$; (B) Análise ultrassonográfica da região supraumbilical direita antes do tratamento, lado controle, $1,74 \mathrm{~cm}$

Fonte: Os autores.

hiperemia após a aplicação, 63\% sentiram leves choques durante a aplicação na região tratada, $4 \%$ sentiram dormência durante a aplicação, $4 \%$ apresentaram irritação na pele, 50\% mencionaram dor durante a aplicação na região lombar e crista ilíaca. Houve relatos somente a respeito da região infraumbilical, ou no modo de aplicação estacionário.

A análise da dor foi feita mediante interpretação do questionário de satisfação aplicado ao término da pesquisa.

Em relação ao questionário de satisfação sobre o tratamento, verificou-se que $63 \%$ notaram as roupas mais folgadas, $38 \%$ perceberam a

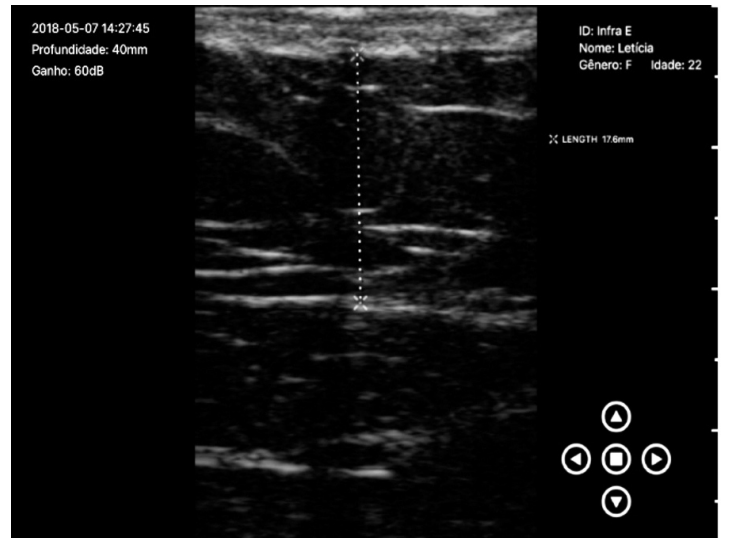

(A)

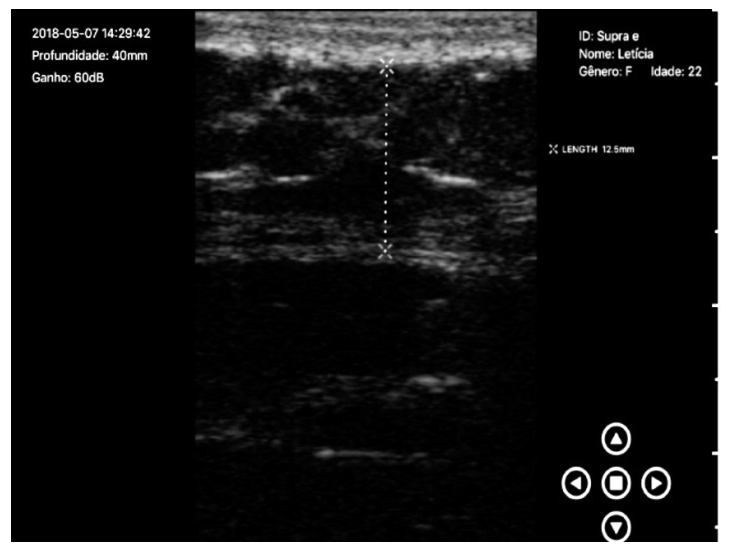

(B)

Figura 5: (A) Anólise ultrassonogrófica da região infraumbilical esquerda, após tratamento, 1,76 cm; (B) Anólise ultrassonográfica da região supraumbilical esquerda, após tratamento, $1,25 \mathrm{~cm}$

Fonte: Os autores.

pele com mais firmeza, $42 \%$ consideraram bom o tratamento, $79 \%$ estavam satisfeitas com o resultado, ocorrendo o relato de melhor resultado no infraumbilical em relação ao supraumbilical.

\section{Discussão}

Observou-se uma redução das medidas de plicometria e na ultrassonografia após as quatro sessões de aplicação da terapia por ondas de choque piezoelétricas. Acredita-se que as ondas mecânicas geram diversos efeitos sobre as células adiposas e modificam o metabolismo celular, 


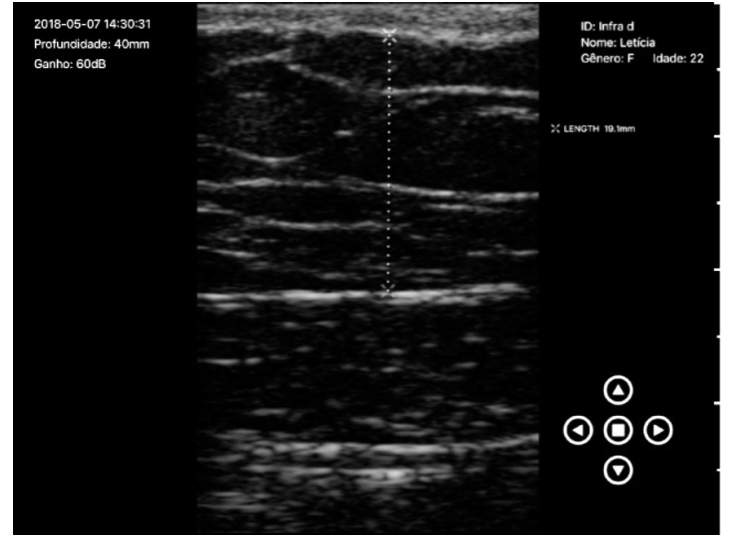

(A)

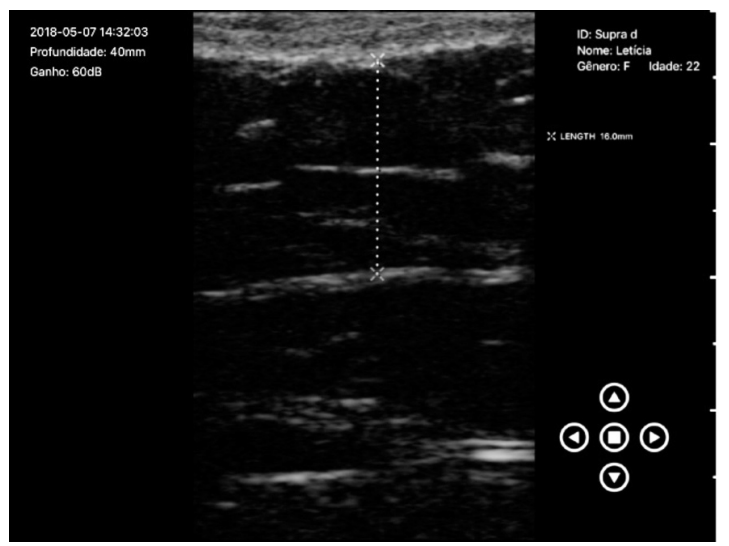

(B)

Figuras 6: (A) Anólise ultrassonogrófica da região infraumbilical direita, após tratamento, lado controle, $1,91 \mathrm{~cm}$; (B) Análise ultrassonográfica da região supraumbilical direita, após tratamento, lado controle, $1,60 \mathrm{~cm}$

Fonte: Os autores.

promovendo um aumento da permeabilidade da parede celular, quebra da molécula de gordura e liberação de triglicerídeos na forma livre associado à regeneração do tecido conjuntivo. Além disso, a TOC pode promover o incremento da microcirculação local com congestão vascular e neoangiogênese, assim como a liberação de óxido nítrico local e do aumento de concentração das prostaglandinas, promovendo a drenagem do excedente de líquido, o que também contribui para o acesso de mediadores químicos que favorecem o processo de degradação da gordura ${ }^{10}$.

A TOC promove um efeito denominado de cavitação, a qual provocaria o rompimento da membrana adiposa por meio da cavitação instável (formação de microbolhas que implodem liberando grande quantidade de energia), sendo assim sugere-se que o tecido adiposo é lesionado por dois tipos de mecanismo: compressão e tração da membrana celular; a qual se repetindo a uma frequência definida promove variação na congruência das moléculas podendo levar ao rompimento das pontes de ligação celular. Sugere-se, também, que, além da ruptura das células adiposas, o efeito da cavitação também provocaria lipólise ${ }^{11-13}$.

A elevação do metabolismo local, provocada pela aplicação das ondas de choque, favorece um aumento da circulação, aumentando a ação de agentes lipolíticos liberados pela indução inflamatória gerada pela ação da TOC. O próprio aumento metabólico favorece um maior consumo energético de modo que se verifica um consumo maior das moléculas de gordura armazenadas na zona de aplicação $0^{14,15}$.

Neste estudo, a perimetria não se alterou, pode-se inferir que isso se deu porque apenas um lado foi tratado; portanto, na avaliação das fotos, não foi observada uma alteração simétrica que promovesse uma redução dessa medida. É possível que a aplicação bilateral tenha promovido a redução dessa medida. Associado a esta resposta, verificou-se que o peso corporal também não sofreu modificações após o tratamento, isto corrobora o objetivo do estudo que era de identificar apenas as mudanças na adiposidade localizada e não na massa corporal total, isso também dá veracidade aos resultados de redução de medida, pois uma grande redução de massa corporal poderia levar a entender que a perda de peso talvez fosse a causa da redução de medidas localizadas.

Na comparação entre as zonas supraumbilical e infraumbilical, foi observada diferença na quantidade de tecido adiposo presente no local, antes da aplicação, não sendo possível fazer uma análise mais independente da aplicação. No entanto, na análise da satisfação e nas respostas adversas após a aplicação, notou-se que as voluntárias apresentaram mais respostas como hiperemia excessiva, irritação, dor e choques na zona de aplicação estacionária da $\mathrm{TOC}^{16-20}$. 
Tabela 5: Distribuição de frequência do questionário de satisfação

\begin{tabular}{|c|c|c|}
\hline & $\begin{array}{c}\text { Frequência } \\
\text { absoluta }\end{array}$ & $\begin{array}{c}\text { Frequência } \\
\text { relativa }\end{array}$ \\
\hline \multicolumn{3}{|l|}{ Notou vermelhidão no local aplicado? } \\
\hline Não & 20 & $88 \%$ \\
\hline Sim & 2 & $8 \%$ \\
\hline Na primeira sessão & 1 & $4 \%$ \\
\hline Em todas as sessões & 1 & $4 \%$ \\
\hline Não & 20 & $88 \%$ \\
\hline \multicolumn{3}{|l|}{ Quanto tempo durou a vermelhidão após aplicação? } \\
\hline Sumiu logo após a primeira sessão em poucos minutos após a aplicação & 21 & $83 \%$ \\
\hline Durou uma hora aproximadamente & 3 & $13 \%$ \\
\hline \multicolumn{3}{|l|}{ Sentiu leves choques durante as aplicações? } \\
\hline Sim & 15 & $63 \%$ \\
\hline Na primeira sessão & 1 & $4 \%$ \\
\hline Nas duas primeiras sessões & 2 & $8 \%$ \\
\hline Em todas as sessões & 5 & $21 \%$ \\
\hline \multicolumn{3}{|l|}{ Notou marcas na pele após a aplicação } \\
\hline Não & 23 & $96 \%$ \\
\hline Na primeira sessão & 1 & $4 \%$ \\
\hline \multicolumn{3}{|l|}{ Quanto tempo duraram as marcas após a aplicação } \\
\hline Sumiu logo após a aplicação & 23 & $96 \%$ \\
\hline Durou um dia & 1 & $4 \%$ \\
\hline \multicolumn{3}{|l|}{ Notou melhora na retenção de líquido/inchaço } \\
\hline Sim & 9 & $33 \%$ \\
\hline Não & 15 & $63 \%$ \\
\hline \multicolumn{3}{|l|}{ Quando percebeu que as roupas ficaram folgadas? } \\
\hline Não & 9 & $33 \%$ \\
\hline Sim & 15 & $63 \%$ \\
\hline Na primeira semana & 1 & $4 \%$ \\
\hline A partir da segunda semana & 4 & $17 \%$ \\
\hline A partir da quarta semana & 2 & $8 \%$ \\
\hline \multicolumn{3}{|l|}{ Em relação à textura da pele, relate sua avaliação } \\
\hline Considero minha pele com mais firmeza agora & 1 & $4 \%$ \\
\hline 3- Considero minha pele com pouca firmeza agora comparado há dois meses & 2 & $8 \%$ \\
\hline / 5- Não visualizei qualquer diferença & 9 & $38 \%$ \\
\hline Considero minha pele mais flácida & 4 & $17 \%$ \\
\hline 3- Considero minha pele com pouca firmeza agora comparada há dois meses & 2 & $8 \%$ \\
\hline / 5- Não visualizei qualquer diferença & 9 & $38 \%$ \\
\hline Considero minha pele mais flácida & 4 & $17 \%$ \\
\hline 3- Considero minha pele com pouca firmeza agora comparada há dois meses & 2 & $8 \%$ \\
\hline \multicolumn{3}{|l|}{ Você ficou satisfeita com o resultado obtido? } \\
\hline Sim & 24 & $79 \%$ \\
\hline Não & 7 & $21 \%$ \\
\hline \multicolumn{3}{|c|}{ Em relação a sua avaliação quanto a este tratamento, descreva o conceito que mais se adequa } \\
\hline Excelente Tratamento & 3 & $13 \%$ \\
\hline Muito bom & 8 & $33 \%$ \\
\hline Bom tratamento & 10 & $42 \%$ \\
\hline Tratamento fraco & 1 & $4 \%$ \\
\hline Não indicaria a ninguém esse tratamento & 1 & $4 \%$ \\
\hline
\end{tabular}


A aplicação da TOC piezoelétrica na forma estacionária apresentou um resultado terapêutico distinto ao da aplicação dinâmica, visto que foi visualizada uma maior redução de tecido adiposo na região em que se usou o modo estacionário. Isso pode ocorrer em virtude de uma maior profundidade de penetração da energia na zona de aplicação no tecido biológico, além de uma maior concentração de energia cavitacional local. Observou-se, ainda, que essa região apresentou um padrão doloroso maior na avaliação subjetiva pelo autorrelato das participantes, gerando desconforto considerável, por isso, recomenda-se para uso clínico a aplicação em dinâmica, na qual, apesar de produzir um resultado menor em relação à redução de adiposidade, resulta em menor intensidade dos sintomas dolorosos e, consequentemente, maior conforto para indivíduo tratado.

Na prática clínica, observa-se, com frequência, que, após a aplicação da terapia de ondas de choque, destaca-se o aumento da sensação dolorosa. É provável que, pelo estímulo tátil gerado nas terminações nervosas locais, ocorra uma intensificação da sensação álgica, principalmente após os primeiros dias de aplicação. No entanto, nesta pesquisa, identificou-se um elevado nível de satisfação quanto aos resultados da intervenção clínica, com percepção de perda de medidas, possibilitando, por exemplo, o uso de roupas de número menor ${ }^{9,17,18,20}$.

Este trabalho apresentou como limitações a ausência de um grupo controle e de outros métodos de análise, como a termografia ou bioimpedância, para verificação do percentual de gordura perdido em cada região. Sugere-se, portanto, a realização de novos estudos em que seja feita uma ampliação dos métodos utilizados para análise de resultados, principalmente o exame histopatológico do tecido tratado.

\section{Conclusão}

A terapia por ondas de choque piezoelétrica, realizada com o modelo de equipamento esco- lhido e na dose proposta neste estudo, promoveu, na amostra estudada, uma redução da adiposidade localizada, constatada nas análises de plicometria e ultrassonografia. Destaca-se que o modo estacionário foi mais efetivo, mas devendo ser evitado pelos efeitos adversos e dores geradas.

\section{Referências}

1. Császár NB, Angstman NB, Milz S, Sprecher CM, Kobel P, Farhat M et al. Radial shock wave devices generate cavitation. Plos One. 2015;10(10).

2. Dymarek R, Bidzińska G, Zwierzchowski K, Słupska L, Ptaszkowski K, Halski T. Evaluation of the effectiveness of extracorporeal shock wave therapy in selected musculoskeletal system disorders of the inflammatory etiology-a critical review of the literature. WiadLek. 2015;68(2):183-92.

3. Hatanaka K, Ito K, Shindo T, Kagaya Y, Ogata T, Eguchi K et al. Molecular mechanisms of the angiogenic effects of low-energy shock wave therapy: roles of mechanotransduction. Am J Physiol Cell Physiol. 2016 Sep 1;311(3):C378-85.

4. Romeo P, Lavanga V, Pagani D, Sansone V. Extracorporeal shock wave therapy in musculoskeletal disorders: a review. Med Princ Pract. 2014;23(1):7-13.

5. Zhang L, Fu XB, Chen S, Zhao ZB, Schmitz C, Weng CS. Efficacy and safety of extracorporeal shock wave therapy for acute and chronic soft tissue wounds: A systematic review and meta-analysis. Int Wound J. 2018 Aug;15(4):590-9.

6. Liao CD, Xie GM, Tsauo JY, Chen HC, Liou TH. Efficacy of extracorporeal shock wave therapy for knee tendinopathies and other soft tissue disorders: a meta-analysis of randomized controlled trials. BMC Musculoskelet Disord. 2018 Aug 2;19(1):278.

7. Siems W, Grune T, Voss P, Brenke R. Antifibrosclerotic effects of shock wave therapy in lipedema and cellulite. Biofactors. 2005;24: 275-82.

8. Zhang Q, Liu L, Sun W, Gao F, Cheng L, Li Z. Extracorporeal shockwave therapy in osteonecrosis of femoral head: a systematic review of now available clinical evidences. Medicine (Baltimore). 2017 Jan;96(4):e5897. 
9. Segot-Chicq E, Compan-Zaouati D, Wolkenstein P, Consoli S, Rodary C, Delvigne V et al. Development and validation of a questionnaire to evaluate how a cosmetic product for oily skin is able to improve well-being in women. J Eur Acad Dermatol Venereol. 2007 Oct;21(9):1181-6.

10. Adatto MA, Adatto-Neilson R, Novak P, Krotz A, Haller G.. Body shaping with acoustic wave therapy AWT®/EPAT®: randomized, controlled study on 14 subjects. J Cosmet Laser Ther. 2011 Dec;13(6):291-6.

11. Braun MT, Daser A, Wroblewska KK. Effects of shock wave therapy on pathological changes in subcutaneous adipose tissue. A pilot study. Aesthet Dermatol. 2005;4:11-7.

12. Koshiyama K1, Kodama T, Yano T, Fujikawa S. Structural change in lipid bilayers and water penetration induced by shock waves: molecular dynamics simulations. Biophys J. 2006 Sep 15;91(6):2198-205.

13. Rinella L, Marano F, Paletto L, Fraccalvieri M, Annaratone L, Castellano I et al. Extracorporeal shock waves trigger tenogenic differentiation of human adipose-derived stem cells. Connect Tissue Res. 2018 Nov; 59(6):561-73.

14. Reznik JE, Biros E, Lamont AC, Sacher Y, Kibrik O, Milanese $S$ et al. A preliminary investigation on the effect of extracorporeal shock wave therapy as a treatment for neurogenic heterotopic ossification following traumatic brain injury. Part I: Effects on pain. Brain Inj. 2017;31(4):526-32.
15. Liang SM, Yeh C-N, Yan J-J, Wan L-R, Lee J-W. In vitro experiment on lysing adipose tissue by shock waves. Journal of Medical and Biological Engineering. 2008;28(4):203-209.

16. Mabrouk MIAI, Badr NM, Taha MM, El Shiwi AMF, Abdelaal AAM, Ali SM, et al. Effect of extracorporeal shock wave therapy on skin blood perfusion in patients with diabetic foot: Randomized controlled trial. Int J Adv Res. 2015;3(3):689-96.

17. Klonschinski T, Ament SJ, Schlereth T, Rompe JD, Birklein F. Application of local anesthesia inhibits effects of low-energy extracorporeal shock wave treatment (ESWT) on nociceptors. Pain Med. 2011 Oct;12(10):1532-7.

18. Skov-Jeppesen SM, Lundrup RN, Ladegaard PBJ, Mortensen J, Sørensen MD, Lund L. Indications and application of extracorporeal shockwave therapy. Ugeskr Laeger. 2019 Feb18;181(8). pii: V07180514.

19. Tang HY, Yu T, Wei W, Zhao Y. Effect of extracorporeal shock wave for tennis elbow: a protocol for systematic review of randomized controlled trial. Medicine (Baltimore). 2019 Feb;98(7):e14517.

20. Wei W, Tang HY, Li YZ, Wang TS. Effectiveness of extracorporeal shock wave for low back pain: a protocol of systematic review. Medicine (Baltimore). 2019 Feb;98(7):e14511. 